\title{
Beyond the Standard Model Higgs Searches at LEP (Part A)
}

\section{Daniel Zer-Zion*}

CERN and University of California Riverside,

CH-1211 Geneva 23, Switzerland

E-mail: Daniel.Zer-Zion@cern.chi

ABstract: Most updated results, obtained by the LEP Collaborations, on searches for Higgs bosons in extensions of the Standard Model are provided. In particular Two Higgs Doublet, Minimal Supersymmetric Standard and Flavour Independent Models are presented. In addition, a new study of the Yukawa process at LEP1 and its implications to the $(g-2)_{\mu}$ is included.

\section{Introduction}

Two Higgs Doublet Models (2HDMs) are attractive extensions of the SM since they add new phenomena with the fewest new parameters; they satisfy the constraints of $\rho \approx 1$ and the absence of tree-level flavour changing neutral currents, if the Higgs-fermion couplings are appropriately chosen. In the context of $2 \mathrm{HDMs}$ the Higgs sector comprises five physical Higgs bosons: two neutral CP-even scalars, $\mathrm{h}^{0}$ and $\mathrm{H}^{0}$ (with $m_{\mathrm{h}}<m_{\mathrm{H}}$ ), one CP-odd scalar, $\mathrm{A}^{0}$, and two charged scalars, $\mathrm{H}^{ \pm}$.

At the centre-of-mass energies accessed by LEP, the $\mathrm{h}^{0}$ and $\mathrm{A}^{0}$ bosons are expected to be produced predominantly via two processes: the Higgs-strahlung process $\mathrm{e}^{+} \mathrm{e}^{-} \rightarrow \mathrm{h}^{0} \mathrm{Z}^{0}$ and the pair-production process $\mathrm{e}^{+} \mathrm{e}^{-} \rightarrow \mathrm{h}^{0} \mathrm{~A}^{0}$. The cross-sections for these two processes, $\sigma_{\mathrm{hZ}}$ and $\sigma_{\mathrm{hA}}$, are related at tree-level to the $\mathrm{SM}$ cross-sections by the following relations [i]1]:

$$
\begin{aligned}
\mathrm{e}^{+} \mathrm{e}^{-} \rightarrow \mathrm{h}^{0} \mathrm{Z}^{0}: & \sigma_{\mathrm{hZ}}=\sin ^{2}(\beta-\alpha) \sigma_{\mathrm{HZ}}^{\mathrm{SM}} \\
\mathrm{e}^{+} \mathrm{e}^{-} \rightarrow \mathrm{h}^{0} \mathrm{~A}^{0}: & \sigma_{\mathrm{hA}}=\cos ^{2}(\beta-\alpha) \bar{\lambda} \sigma \mathrm{SM}_{\mathrm{HZ}}
\end{aligned}
$$

where $\sigma_{\mathrm{HZ}}^{\mathrm{SM}}$ is the Higgs-strahlung cross-section for the SM process $\mathrm{e}^{+} \mathrm{e}^{-} \rightarrow \mathrm{H}_{\mathrm{SM}}^{0} \mathrm{Z}^{0}$, and $\bar{\lambda}$ is a phase-space factor.

${ }^{*}$ Speaker. 
The coefficients $\sin ^{2}(\beta-\alpha)$ and $\cos ^{2}(\beta-\alpha)$ which appear in Eqs. (1) and (1) (1) determine the production cross-sections. The decay branching ratios to the various final states are also determined by $\alpha$ and $\beta$. In the $2 \mathrm{HDM}(\mathrm{II})$ the tree-level couplings of the $\mathrm{h}^{0}$ and $\mathrm{A}^{0}$ bosons to the up- and down-type quarks relative to the canonical SM values are [i]

$$
\mathrm{h}^{0} \mathrm{c} \overline{\mathrm{c}}: \frac{\cos \alpha}{\sin \beta}, \quad \mathrm{h}^{0} \mathrm{~b} \overline{\mathrm{b}}:-\frac{\sin \alpha}{\cos \beta}, \quad \mathrm{A}^{0} \mathrm{c} \overline{\mathrm{c}}: \cot \beta, \quad \mathrm{A}^{0} \mathrm{~b} \overline{\mathrm{b}}: \tan \beta .
$$

The results on the scanning of the $2 \mathrm{HDM}$ parameter space are presented in Section

The Minimal Supersymmetric Standard Model (MSSM) predicts the existence of two complex scalar field doublets, if the Higgs sector of the MSSM is assumed to conserve CP the physical Higgs bosons are the CP-even $\mathrm{h}^{0}$ and $\mathrm{H}^{0}$, the $\mathrm{CP}$-odd $\mathrm{A}^{0}$, and the charged

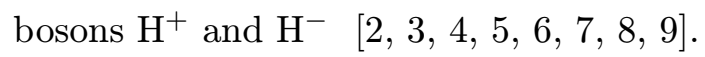

In the MSSM, the Higgs-strahlung process $\mathrm{e}^{+} \mathrm{e}^{-} \rightarrow \mathrm{h}^{0} \mathrm{Z}^{0}$ proceeds as in the Standard Model, but its rate is suppressed by the factor $\sin ^{2}(\beta-\alpha)$. The latest LEP-Wide MSSM results are presented in Section $\overline{3}$.

Higgs bosons couple to fermions with a strength proportional to the fermion mass, favouring the decays into pairs of b-quarks and tau leptons at LEP energies. However, with values of $\alpha$ and $\tan \beta$ close to zero the decays into up-type light quarks and gluons through quark loops become dominant, motivating the inclusion of flavour independent analyses.

All four LEP collaborations have pursued such flavour independent searches in recent

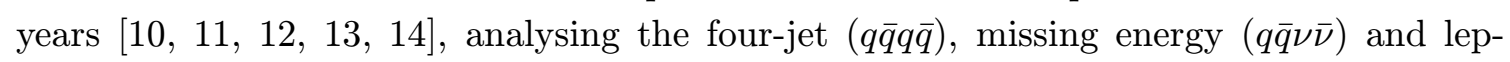
tonic $\left(q \bar{q} l^{+} l^{-}\right)$topologies. None has found evidence for any signal. The first LEP-wide combination of the results obtained is presented in Section

In non-supersymmetric $2 \mathrm{HDM}$, Higgs bosons with small masses still cannot be ex-

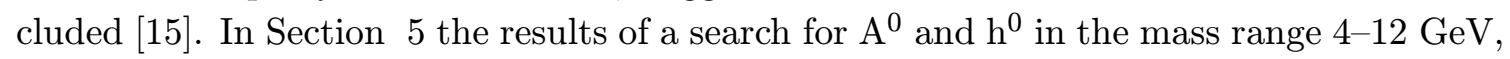
using LEP1 data, are presented, togetehr wih new constraints on the parameter space of these models and its implications for the $(g-2)_{\mu}$ using a search for the Yukawa Process at LEP1.

\section{Two Higgs Doublet Model Interpretation}

The interpretation of the searches for the neutral Higgs bosons in the $2 \mathrm{HDM}(\mathrm{II})$ is done by scanning the parameter space of the model. Every $\left(m_{\mathrm{h}}, m_{\mathrm{A}}, \tan \beta, \alpha\right)$ point determines the production cross-section and the branching ratios to different final states.

The parameter space covered by the OPAL study [i] $\overline{0} \overline{0}$ is: $1 \leq m_{\mathrm{h}} \leq 120 \mathrm{GeV}, 3 \mathrm{GeV}$ $\leq m_{\mathrm{A}} \leq 2.0 \mathrm{TeV}, 0.4 \leq \tan \beta \leq 58.0$, and $\alpha=\pi / 2, \pi / 4,0,-\pi / 4$, and $-\pi / 2$. The values of $\alpha$ are chosen to extend the analysis to the particular cases of maximal and minimal mixing in the neutral CP-even sector of the $2 \mathrm{HDM}$ (II) $(\alpha= \pm \pi / 4$ and $\pm \pi / 2$, respectively) and of $\mathrm{BR}\left(\mathrm{h}^{0} \rightarrow \mathrm{b} \overline{\mathrm{b}}\right)=0(\alpha=0)$. The extreme cases $\alpha= \pm \pi / 2$ are equivalent in the $2 \mathrm{HDM}(\mathrm{II})$ since the mass matrix of the CP-even neutral Higgs sector, containing the Higgs doublets, becomes diagonal. In addition any two $\alpha$ values separated by a $\pi$ interval represent the same $2 \mathrm{HDM}(\mathrm{II})$. 
In Figures $\mathrm{I}_{1}^{1}(\mathrm{a}-\mathrm{d})$ the excluded regions in the $\left(m_{\mathrm{h}}, m_{\mathrm{A}}\right)$ plane are shown for the chosen values of $\alpha$, together with the calculated expected exclusion limits. A particular $\left(m_{\mathrm{h}}, m_{\mathrm{A}}\right.$, $\alpha)$ point is excluded at $95 \%$ CL if it is excluded for all scanned values of $\tan \beta$. Different domains of $\tan \beta$ are studied: a) $0.4 \leq \tan \beta \leq 58.0$ and b) $0.4 \leq \tan \beta \leq 1.0$ or $1.0<\tan \beta \leq$ 58.0, for which enlarged excluded regions are obtained.

In Figure $2_{-1}^{2}$ the excluded regions in the $\left(m_{\mathrm{h}}, m_{\mathrm{A}}\right)$ plane independent of $\alpha$ are given together with the calculated expected exclusion limits. A particular $\left(m_{\mathrm{h}}, m_{\mathrm{A}}\right)$ point is excluded at 95\% CL if it is excluded for all scanned values of $\tan \beta$ and $\alpha$. Different domains of $\tan \beta$ are shown: $0.4 \leq \tan \beta \leq 58.0$ (darker grey area), $0.4 \leq \tan \beta \leq 1.0$ (lighter grey area) and $1.0<\tan \beta \leq 58.0$ (hatched area), for which enlarged excluded regions are obtained. A rectangular region $1 \lesssim m_{\mathrm{h}} \lesssim 58 \mathrm{GeV}$ for $10 \lesssim m_{\mathrm{A}} \lesssim 65 \mathrm{GeV}$ is fully excluded at 95\% CL independent of $\alpha$ and $\tan \beta$. The cross-hatched region shows the exclusion provided by the constraints on the width of the $\mathrm{Z}^{0}$ common to all the scanned values of $\alpha$ and $\tan \beta$.

\section{Limits in the MSSM Parameter Space}

\subsection{Benchmark Scenarios}

Three benchmark scenarios are considered. The first ("no-mixing" scenario) assumes that there is no mixing between the scalar partners of the left-handed and the right-handed top quarks, with the following values and ranges for the parameters: $\mathrm{M}_{\mathrm{SUSY}}=1 \mathrm{TeV} / c^{2}$, $M_{2}=200 \mathrm{GeV} / c^{2}, \mu=-200 \mathrm{GeV} / c^{2}, X_{t}(\equiv A-\mu \cot \beta)=0,0.4<\tan \beta<50$ and $4 \mathrm{GeV} / c^{2}<m_{\mathrm{A}}<1 \mathrm{TeV} / c^{2}$. The gluino mass $\mathrm{m}_{\tilde{\mathrm{g}}}$ is set to $800 \mathrm{GeV} / c^{2}$; it has little effect on the phenomenology of this scenario. The assumption that the decay widths can be neglected is only valid for $\tan \beta<30$ in this scenario, and hence higher values of $\tan \beta$ are not considered.

The second scenario (" $m_{\mathrm{h} 0}-\max$ ") is designed to yield the maximal value of $m_{\mathrm{h}}$ in the model. The $m_{\mathrm{h}^{0}}-$ max scenario corresponds to the most conservative range of excluded $\tan \beta$ values for fixed values of the mass of the top quark and $\mathrm{M}_{\mathrm{SUSY}}$. The values of the parameters in the $m_{\mathrm{h}^{0}}$-max scenario are fixed at the same values used in the no-mixing scenario, except for the stop mixing parameter $X_{t}=2 \mathrm{M}_{\mathrm{SUSY}}$, using the conventions of the two-loop diagrammatic calculation of $\left[\mathbb{8}_{1}^{1}, 1\right]$ in this model also in order to satisfy the assumptions made on the decay widths.

The third scenario ("large $\mu$ " scenario) is a scan with parameters chosen to be $\mathrm{M}_{\mathrm{SUSY}}=$ $400 \mathrm{GeV} / c^{2}, \mu=1 \mathrm{TeV} / c^{2}, M_{2}=400 \mathrm{GeV} / c^{2}, \mathrm{~m}_{\tilde{\mathrm{g}}}=200 \mathrm{GeV} / c^{2}, 4 \leq m_{\mathrm{A}} \leq 400 \mathrm{GeV} / c^{2}$, $X_{t}=-300 \mathrm{GeV} / c^{2}$. This scenario is designed to illustrate choices of MSSM parameters for which the Higgs boson $\mathrm{h}^{0}$ does not decay into pairs of $\mathrm{b}$ quarks due to large corrections from SUSY loop processes. In this scenario, for all choices of $m_{\mathrm{A}}$ and $\tan \beta$, at least one Higgs boson signal with a large production cross-section is within the kinematic reach of LEP2. The maximum value of $m_{\mathrm{h}}$ in this scenario is slightly less than $108 \mathrm{GeV} / c^{2}$.

The 95\% CL exclusion contours are shown in Figure $\underline{3}_{1}$ for the $m_{\mathrm{h}^{0}}-$ max scenario, and in Figure i⿱⺈ $_{-1}$ for the no-mixing scenario. In the no-mixing and $m_{\mathrm{h}^{0}}$-max scenarios, limits are shown in four projections: the $\left(m_{\mathrm{h}}, m_{\mathrm{A}}\right)$ projection, the $\left(m_{\mathrm{h}}, \tan \beta\right)$ projection, the 


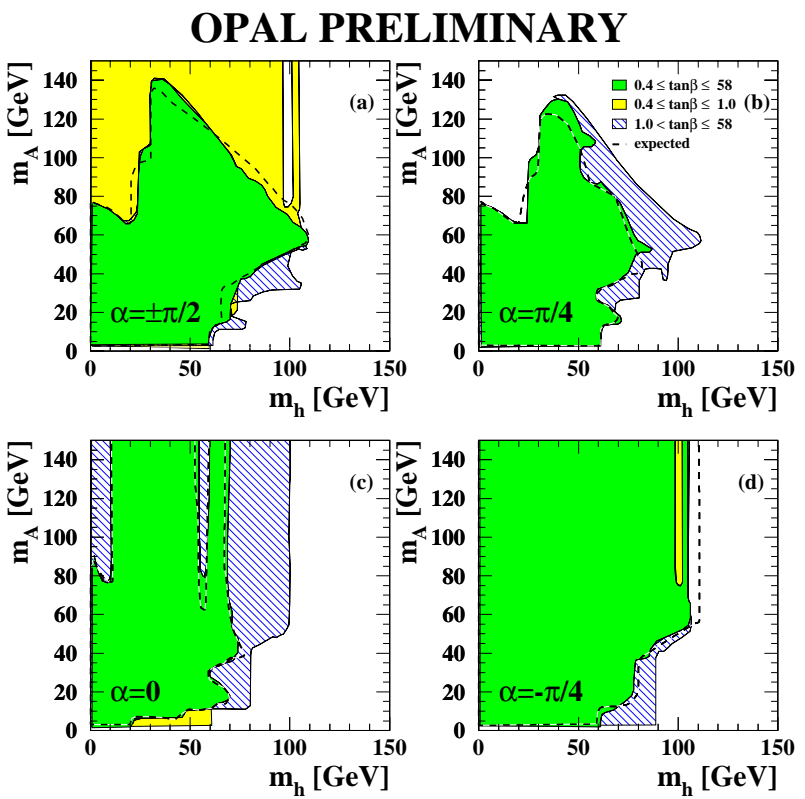

Figure 1: Excluded regions in the $\left(m_{\mathrm{h}}, m_{\mathrm{A}}\right)$ plane, (a)-(d), for $\alpha= \pm \pi / 2, \pi / 4, \quad 0$ and $-\pi / 4$, respectively, together with the expected exclusion limits. A particular $\left.\left(m_{\mathrm{h}}, m_{\mathrm{A}}\right), \alpha\right)$ point is excluded at $95 \% \mathrm{CL}$ if it is excluded for all scanned values of $\tan \beta$. Three different domains of $\tan \beta$ are shown: the darker grey region is excluded for all values $0.4 \leq \tan \beta \leq 58.0$; additional enlarged excluded regions are obtained constraining 0.4 $\leq \tan \beta \leq 1.0$ (lighter grey area) or $1.0<$ $\tan \beta \leq 58.0$ (hatched area). Expected exclusion limits are shown for $0.4 \leq \operatorname{tanb} \leq 58.0$ (dashed line).

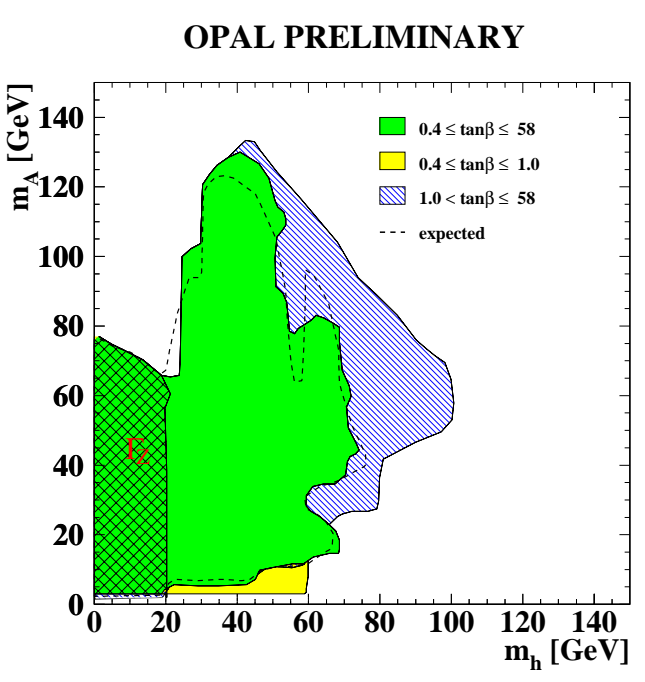

Figure 2: Excluded $\left(m_{\mathrm{h}}, m_{\mathrm{A}}\right)$ region independent of $\alpha$, together with the expected exclusion limit. A particular $\left(m_{\mathrm{h}}, m_{\mathrm{A}}\right)$ point is excluded at $95 \% \mathrm{CL}$ if it is excluded for $0.4 \leq \tan \beta \leq 58.0$ (darker grey region), 0.4 $\leq \tan \beta \leq 1.0$ (lighter grey region) and 1.0 $<\tan \beta \leq 58.0$ (hatched region) for $-\pi / 2 \leq$ $\alpha \leq \pi / 2$. The cross-hatched region is excluded using constraints from $\Gamma_{Z}$ only. Expected exclusion limits are shown as a dashed line.

$\left(m_{\mathrm{A}}, \tan \beta\right)$ projection, and the $\left(m_{\mathrm{H}^{ \pm}}, \tan \beta\right)$ projection. The observed and expected limits for $m_{\mathrm{h}}$ and $m_{\mathrm{A}}$ for the $m_{\mathrm{h}^{0}}-\max$ and no-mixing scenarios are given in Table

The combination of the four experiments' results in the large $\mu$ scenario now includes

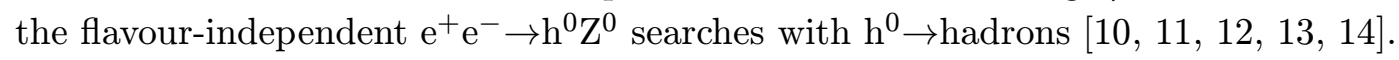

The previous combination [i] $[\overline{8} \overline{8}]$ of the LEP search results, which only included channels relying on $\mathrm{h}^{0}$ decays to $\mathrm{b} \overline{\mathrm{b}}$ and to $\tau^{+} \tau^{-}$, did not exclude some choices of $m_{\mathrm{A}}$ and $\tan \beta$. Because the light Higgs boson $\mathrm{h}^{0}$ has a mass less than $108 \mathrm{GeV} / c^{2}$ for all choices of $\left(m_{\mathrm{A}}, \tan \beta\right)$ in this model, and because $\mathrm{e}^{+} \mathrm{e}^{-} \rightarrow \mathrm{H}^{0} \mathrm{Z}^{0}$ is within kinematic reach whenever $\mathrm{e}^{+} \mathrm{e}^{-} \rightarrow \mathrm{h}^{0} \mathrm{Z}^{0}$ is suppressed by a small $\sin ^{2}(\beta-\alpha)$ and $m_{\mathrm{h}}+m_{\mathrm{A}}>\sqrt{s}$, there is always a Higgs signal with sizeable strength for all considered models within the large $\mu$ scenario. The challenge of this scenario is to test models with non-b $\bar{b}$ and non- $\tau^{+} \tau^{-}$decay modes. 


\begin{tabular}{|c|c|c|c|}
\hline Scenario & $m_{\mathrm{h}}$ limit $\left(\mathrm{GeV} / c^{2}\right)$ & $m_{\mathrm{A}}$ limit $\left(\mathrm{GeV} / c^{2}\right)$ & $\begin{array}{c}\text { Excluded } \tan \beta \\
\text { observed limit (expected limit) }\end{array}$ \\
\hline$m_{\mathrm{h} 0}-\max$ & $91.0(94.6)$ & $91.9(95.0)$ & $0.5<\tan \beta<2.4(0.5<\tan \beta<2.6)$ \\
No Mixing & $91.5(95.0)$ & $92.2(95.3)$ & $0.7<\tan \beta<10.5(0.8<\tan \beta<16.0)$ \\
\hline
\end{tabular}

Table 1: Limits on $m_{\mathrm{h}}$ and $m_{\mathrm{A}}$ in the $m_{\mathrm{h}^{0}}-$ max and no-mixing benchmark scenarios explained in the text. The median expected limits in an ensemble of SM background-only experiments are listed in parentheses. To highlight the sensitivity of the searches for massive Higgs bosons, the limits on $m_{\mathrm{h}}$ and $m_{\mathrm{A}}$ are given with the additional constraint of $\tan \beta>0.7$ for the no-mixing scenario. If $\tan \beta$ is explored in the full region to 0.4 , then values of $m_{\mathrm{A}}$ below $40 \mathrm{GeV} / c^{2}$ are not excluded for values of $m_{\mathrm{h}}$ above $65 \mathrm{GeV} / c^{2}$ in the no-mixing scenario.

A careful scan over the model space indicates that the addition of the flavour-independent $\mathrm{e}^{+} \mathrm{e}^{-} \rightarrow \mathrm{h}^{0} \mathrm{Z}^{0}$ searches adds enough sensitivity to exclude the models which were previously unexcluded, although for some model points they are interpreted as flavour-independent $\mathrm{e}^{+} \mathrm{e}^{-} \rightarrow \mathrm{H}^{0} \mathrm{Z}^{0}$ searches. This scenario is therefore entirely excluded at the $95 \%$ confidence level.

\subsection{Coupling Strength Limits}

More stringent limits on the $\mathrm{h}^{0} \mathrm{~A}^{0}$ production cross-section allow tests of models which either predict lower cross-sections or reduced branching ratios of the Higgs bosons to the final states which are sought at LEP. Examples of such models are those involving substantial CP-violation in the MSSM Higgs sector and general two-Higgs-doublet models (2HDMs) without SUSY constraints.

The $\mathrm{e}^{+} \mathrm{e}^{-} \rightarrow \mathrm{h}^{0} \mathrm{~A}^{0}$ search results from the four experiments are combined using the MSSM model considered in the $m_{\mathrm{h}^{0}}-$ max scenario above to compute the dependence of the $\mathrm{e}^{+} \mathrm{e}^{-} \rightarrow \mathrm{h}^{0} \mathrm{~A}^{0}$ cross-section on the centre-of-mass energy. The coupling limits are produced separately for the set of $\mathrm{h}^{0} \mathrm{~A}^{0} \rightarrow \mathrm{b} \overline{\mathrm{b}} \mathrm{b}$ search channels, for the set of $\mathrm{h}^{0} \mathrm{~A}^{0} \rightarrow \mathrm{b} \bar{b} \tau^{+} \tau^{-}$search channels, and also for a combination of all $\mathrm{h}^{0} \mathrm{~A}^{0}$ search channels assuming fixed branching ratios. The branching ratios chosen for the third set of coupling limits is $\operatorname{Br}\left(\mathrm{h}^{0} \rightarrow \mathrm{b} \overline{\mathrm{b}}\right)=0.94$, $\operatorname{Br}\left(\mathrm{A}^{0} \rightarrow \mathrm{b} \bar{b}\right)=0.92, \operatorname{Br}\left(\mathrm{h}^{0} \rightarrow \tau^{+} \tau^{-}\right)=0.06$ and $\operatorname{Br}\left(\mathrm{A}^{0} \rightarrow \tau^{+} \tau^{-}\right)=0.08$, which are typical in the $m_{\mathrm{h}^{0}}-$ max scenario for values of $\tan \beta$ greater than 10 . In all cases, $\cos ^{2}(\beta-\alpha)=1$, and the signal is multiplied by a scale factor such that the scaled signal is excluded at exactly the $95 \%$ confidence level $\left(\mathrm{CL}_{\mathrm{s}}=0.05\right)$. Presently, no $\mathrm{h}^{0} \mathrm{~A}^{0} \rightarrow \tau^{+} \tau^{-} \tau^{+} \tau^{-}$searches are combined. The coupling strength limits for the three combinations of channels are shown in Figures ${ }_{-1}^{-5}$ and $\overline{\underline{b}}_{1}^{1}$. These limits can be interpreted as upper bounds on $\cos ^{2}(\beta-$ $\alpha) \mathrm{B} r\left(\mathrm{~h}^{0} \rightarrow \mathrm{b} \overline{\mathrm{b}}\right) \mathrm{B} r\left(\overline{\mathrm{A}}^{0} \rightarrow \mathrm{b} \overline{\mathrm{b}}\right), \cos ^{2}(\beta-\alpha) \mathrm{B} r\left(\mathrm{~h}^{0} \rightarrow \mathrm{b} \overline{\mathrm{b}}\right) \mathrm{B} r\left(\mathrm{~A}^{0} \rightarrow \tau^{+} \tau^{-}\right)$, and $\cos ^{2}(\beta-\alpha)$ assuming the fixed branching ratios mentioned above. The limits for the $\mathrm{b} \overline{\mathrm{b}} \tau^{+} \tau^{-}$channels can be interpreted for either the $\mathrm{h}^{0}$ or the $\mathrm{A}^{0}$ decaying into $\tau^{+} \tau^{-}$, while the other decays to $\mathrm{b} \overline{\mathrm{b}}$. The experimental limits are shown in the figures along with the limits that are expected in an ensemble of hypothetical experiments in which there is no signal. 

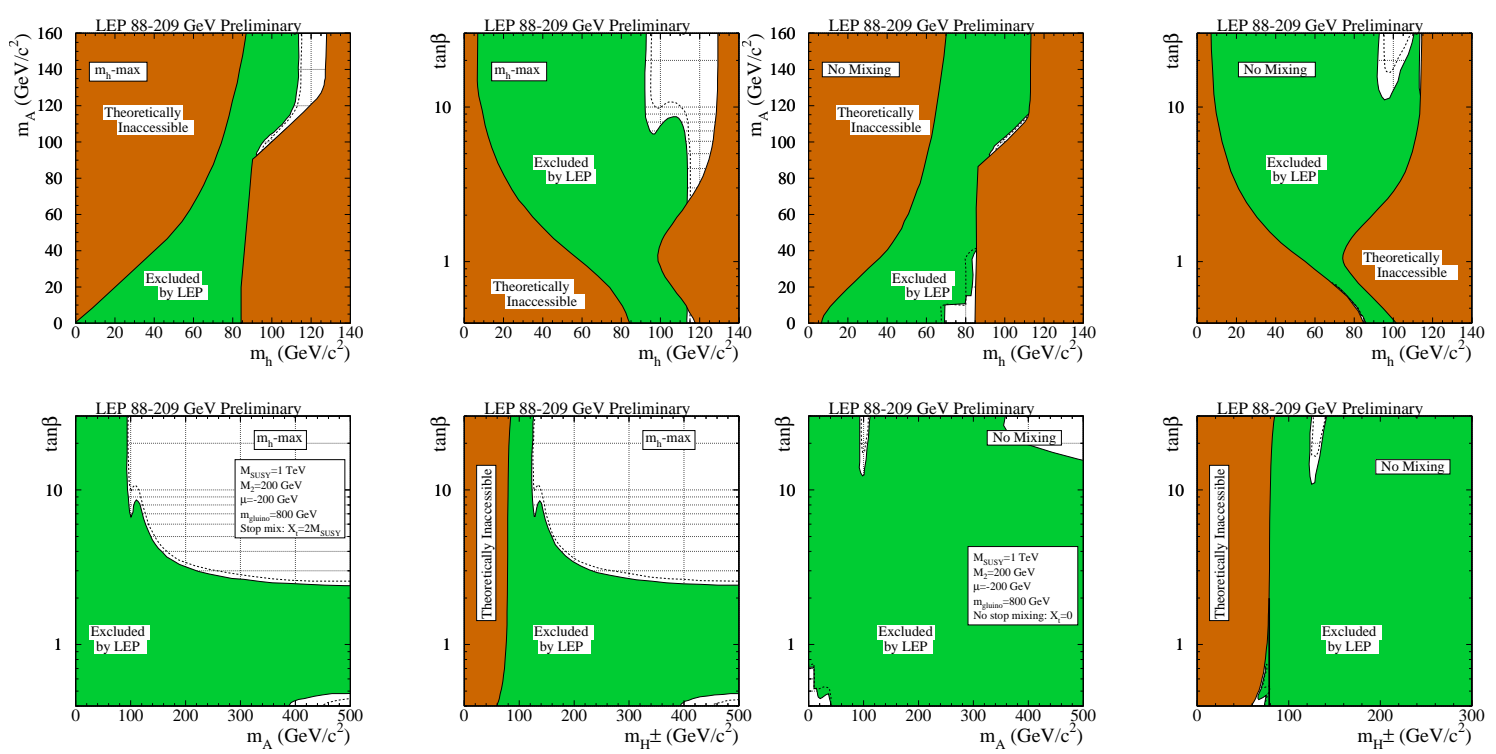

Figure 3: The MSSM exclusion for the $m_{\mathrm{h}^{-}}$ max benchmark scenario. This figure shows the excluded (diagonally hatched) and theoretically disallowed (cross-hatched) regions as functions of the MSSM parameters in four projections: (upper left) the $\left(m_{\mathrm{h}}, m_{\mathrm{A}}\right)$ plane, (upper right) the $\left(m_{\mathrm{h}}, \tan \beta\right)$ plane, (lower left) the $\left(m_{\mathrm{A}}, \tan \beta\right)$ plane and (lower right) the $\left(m_{\mathrm{H}^{ \pm}}, \tan \beta\right)$ plane. The dashed lines indicate the boundaries of the regions expected to be excluded at the $95 \%$ CL if only SM background processes are present.

Figure 4: The MSSM exclusion for the "nomixing" benchmark scenario. This figure shows the excluded (diagonally hatched) and theoretically inaccessible (cross-hatched) regions as functions of the MSSM parameters in four projections: (upper left) the $\left(m_{\mathrm{h}}, m_{\mathrm{A}}\right)$ plane, (upper right) the $\left(m_{\mathrm{h}}, \tan \beta\right)$ plane, (lower left) the $\left(m_{\mathrm{A}}, \tan \beta\right)$ plane and (lower right) the $\left(m_{\mathrm{H}^{ \pm}}, \tan \beta\right)$ plane. The dashed lines indicate the boundaries of the regions expected to be excluded at the $95 \%$ CL if only SM background processes are present.

\section{Flavour Independent Searches}

The common evaluation of the results used the standard statistical procedures based on the likelihood ratio technique, as applied in the other combinations performed by the LEP working group for Higgs boson searches.

Results were first obtained for each of the four collaborations, with two independent implementations of the combination software, and compared with the results obtained within each collaboration. The general shapes and features of the observed and expected confidence levels for the signal, $C L_{s}$ and background-only, $C L_{b}$ hypotheses were very consistent.

The confidence levels $C L_{s}$ and $C L_{b}$ obtained from the full combination in the signal and background-only hypotheses are shown as a function of the mass in Figure 17 A 5 sigma discovery corresponds to a value of $5.710^{-7}$, as indicated by the horizontal line. The maximal sensitivity for such a discovery is reached for an assumed Higgs boson mass of $107 \mathrm{GeV} / \mathrm{c}^{2}$, when this line is intersected by the expected median confidence in the background-only hypothesis. 


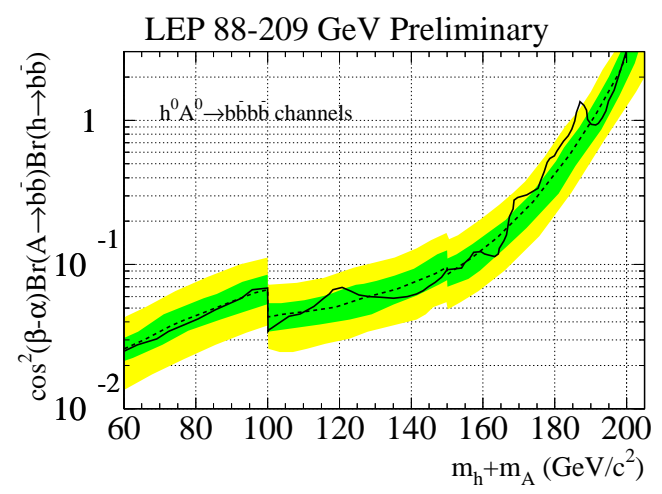

Figure 5: Limits on $\cos ^{2}(\beta-\alpha)$ $\operatorname{Br}\left(\mathrm{h}^{0} \rightarrow \mathrm{b} \overline{\mathrm{b}}\right) \operatorname{Br}\left(\mathrm{A}^{0} \rightarrow \mathrm{b} \overline{\mathrm{b}}\right), \quad$ assuming $\quad m_{\mathrm{h}} \approx$ $m_{\mathrm{A}}$, and the energy-dependence of the $\mathrm{e}^{+} \mathrm{e}^{-} \rightarrow \mathrm{h}^{0} \mathrm{~A}^{0} \quad$ cross-section from the $m_{\mathrm{h}^{0}}-\max$ scenario. The solid line is the observed limit, and the dashed line is the median expected limit in an ensemble of hypothetical experiments in the absence of a signal. Contours indicating the $68 \%$ and $95 \%$ probability bands centred on the median expectation show the expected variation of the limit in an ensemble of background-only experiments.

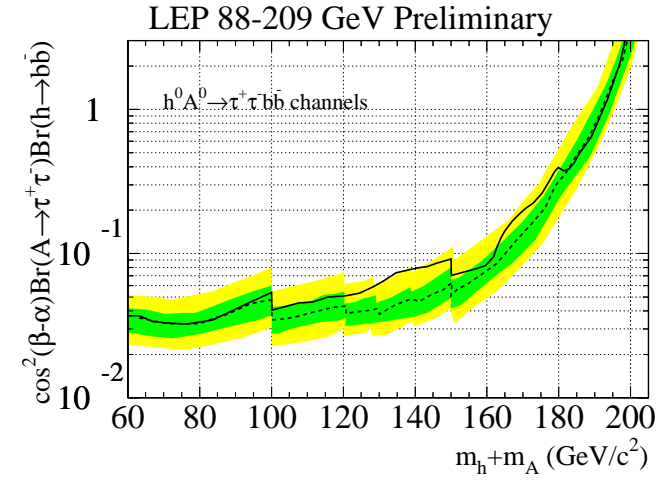

Figure 6: Limits on $\cos ^{2}(\beta-\alpha)$ $\operatorname{Br}\left(\mathrm{h}^{0} \rightarrow \mathrm{b} \bar{b}\right) \operatorname{Br}\left(\mathrm{A}^{0} \rightarrow \tau^{+} \tau^{-}\right), \quad$ assuming $m_{\mathrm{h}} \approx m_{\mathrm{A}}$ and the energy-dependence of the $\mathrm{e}^{+} \mathrm{e}^{-} \rightarrow \mathrm{h}^{0} \mathrm{~A}^{0}$ cross-section from the $m_{\mathrm{h}^{0}}-\max$ scenario. The solid line is the observed limit, and the dashed line is the median expected limit in an ensemble of hypothetical experiments in the absence of a signal. Contours indicating the $68 \%$ and $95 \%$ probability bands centred on the median expectation show the expected variation of the limit in an ensemble of background-only experiments.

The combined exclusion from the four collaborations is shown as a function of the mass in Figure 'si:

\section{Implications of the Yukawa Process to the $(g-2)_{\mu}$}

The cross-section of the Yukawa process [1] 190.1$], \sigma_{\text {Yukawa }} \propto m_{\mathrm{f}}^{2} N_{\mathrm{c}} \xi_{\mathrm{f}}^{2}$, is proportional to the squared fermion mass, $m_{\mathrm{f}}^{2}$, the colour factor, $N_{\mathrm{c}}$, of the emitting fermion, and an enhancement factor $\xi_{\mathrm{f}}^{2}$, which describes the coupling between the Higgs boson and the emitting fermion.

The range of $\xi_{d}^{\mathrm{h} / \mathrm{A}}$ for which a detectable signal would be produced can be divided

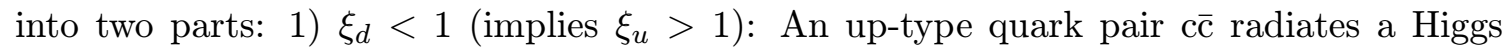
boson decaying into $c \bar{c}$, and 2) $\xi_{d}>1$ : A down-type quark pair b $\bar{b}$ radiates a Higgs boson decaying into $\tau^{+} \tau^{-}$.

Light Higgs bosons $\mathrm{A}^{0}$ and $\mathrm{h}^{0}$ could form a part of $a_{\mu}$ via loop diagrams. A one-loop calculation [i2 $\overline{0}]$ predicts positive contributions $\delta a_{\mu}^{I}(\mathrm{~h})>0$ for $\mathrm{h}^{0}$, and negative contributions $\delta a_{\mu}^{I}(\mathrm{~A})<0$ for $\mathrm{A}^{0}$. The two-loop terms, due to the stronger coupling of the Higgs fields to loops with heavy quarks, turn out to be larger in magnitude than the one-loop terms, and of opposite sign [2]1] , giving a total positive contribution $\delta a_{\mu}^{I I}(\mathrm{~A})>0$ for the $\mathrm{A}^{0}$, as shown with indicated isolines in Figure $\overline{9}_{1}(\mathrm{a})[\overline{2} \overline{2} \overline{2}]$. However, the two-loop terms gives a 


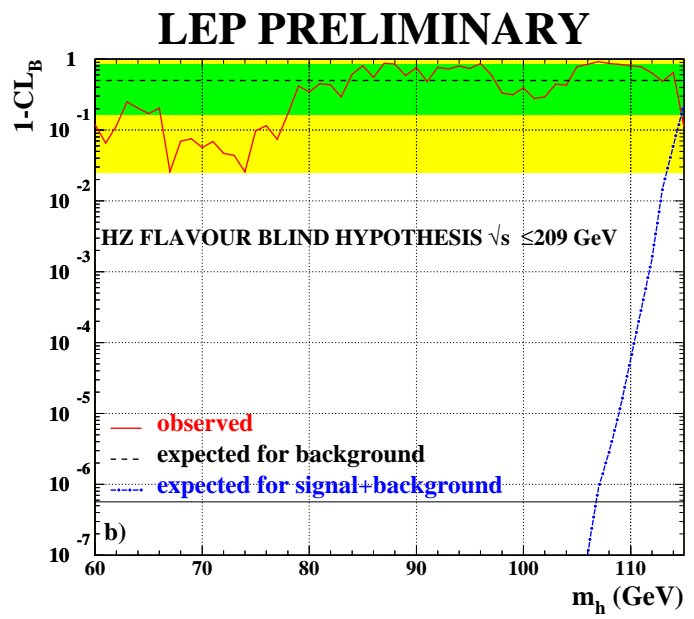

Figure 7: Combined LEP confidence levels as a function of the Higgs boson mass in the flavour independent hypothesis, assuming production cross-sections equal to those in the Standard Model and $\mathrm{BR}(\mathrm{h} \rightarrow$ hadrons $)$ $=1.0$. The confidence levels for the signal and background-only hypotheses are shown in the plot. The curves are the observed (solid) and expected median (dashed) confidences from background-only experiments, and the bands are the corresponding 68.3 $\%$ and $95 \%$ confidence intervals. The dotdashed line shows the expected median confidence from experiments including an expected signal of mass given in abscissa.

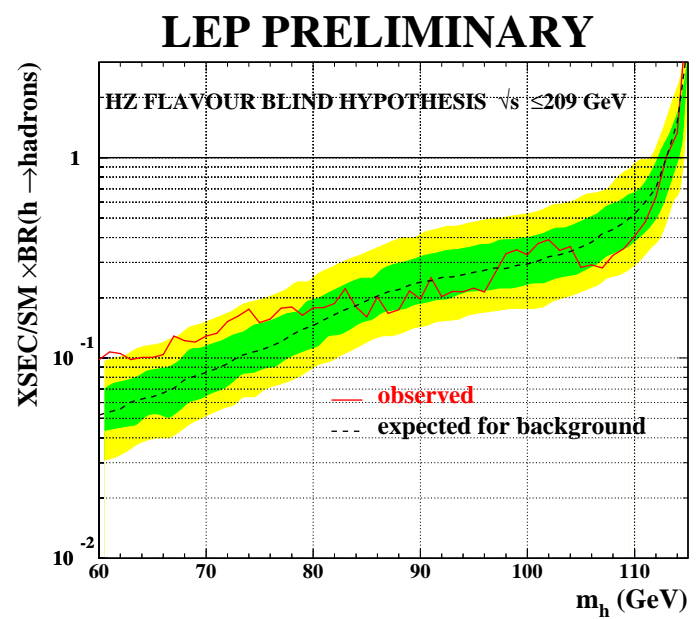

Figure 8: Combined flavour independent LEP observed and expected 95\% CL upper limits on the production cross-section as a function of the Higgs boson mass, normalised to the expected Standard Model values, computed assuming $\mathrm{BR}(\mathrm{h} \rightarrow$ hadrons $)=1.0$. The curves are the observed (solid) and expected median (dashed) excluded ratios, and the bands correspond to $68.3 \%$ and $95 \%$ confidence intervals from the background-only experiments.

total negative contribution $\delta a_{\mu}^{I I}(\mathrm{~h})<0$ for the $\mathrm{h}^{0}$, thus suggesting that the $\mathrm{h}^{0}$ can not account for the BNL observation. In Figure ${ }_{1-1}^{-1}(\mathrm{~b})$ only the isolines of the contribution from the earlier one-loop calculation [23] which resulted in a positive value of $\delta a_{\mu}^{I}(\mathrm{~h})$ are shown. The data exclude positive contributions $\delta a_{\mu}^{I}(\mathrm{~h})>100 \times 10^{-11}$ for h masses between 4.0 and $10.7 \mathrm{GeV}$ at the one-loop level, and $\delta a_{\mu}^{I I}(\mathrm{~A})>100 \times 10^{-11}$ for A masses betwen 4.0 and $9.9 \mathrm{GeV}$ at the two-loop level. Similar limits have been derived from radiative

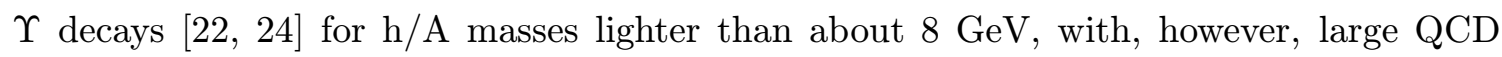
uncertainies.

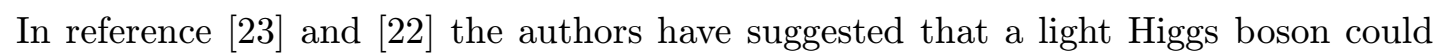
fully account for the observed deviation of the measured $(g-2)_{\mu}$ from the Standard Model expectation. In a scenario without contributions of other new particles, eg. gauginos, and assuming that either $\mathrm{h}^{0}$ or $\mathrm{A}^{0}$ is heavy enough to render associated $\mathrm{A}^{0} \mathrm{~h}^{0}$ production inaccessible at LEP, only the lighter of the two Higgs bosons would sizeably contribute. For a light $\mathrm{h}^{0}$, one has to assume in addition $\sin (\beta-\alpha) \approx 0$ to explain its non-observation in the Standard Model search for the Higgsstrahlung process.

The experimental rule out (using the one-loop calculation [203]) a light $\mathrm{h}^{0}$ in the mass 
range of $4-10.7 \mathrm{GeV}$, and (using the two-loop calculation [20 $\left.{ }_{2}^{2} \overline{1}_{1}\right)$ a light $\mathrm{A}^{0}$ in the mass range from $4-9.9 \mathrm{GeV}$ as the only source of the discrepancy in the $(g-2)_{\mu}$ measurement for all

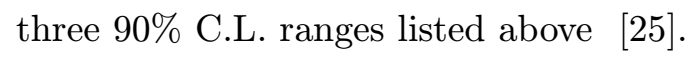

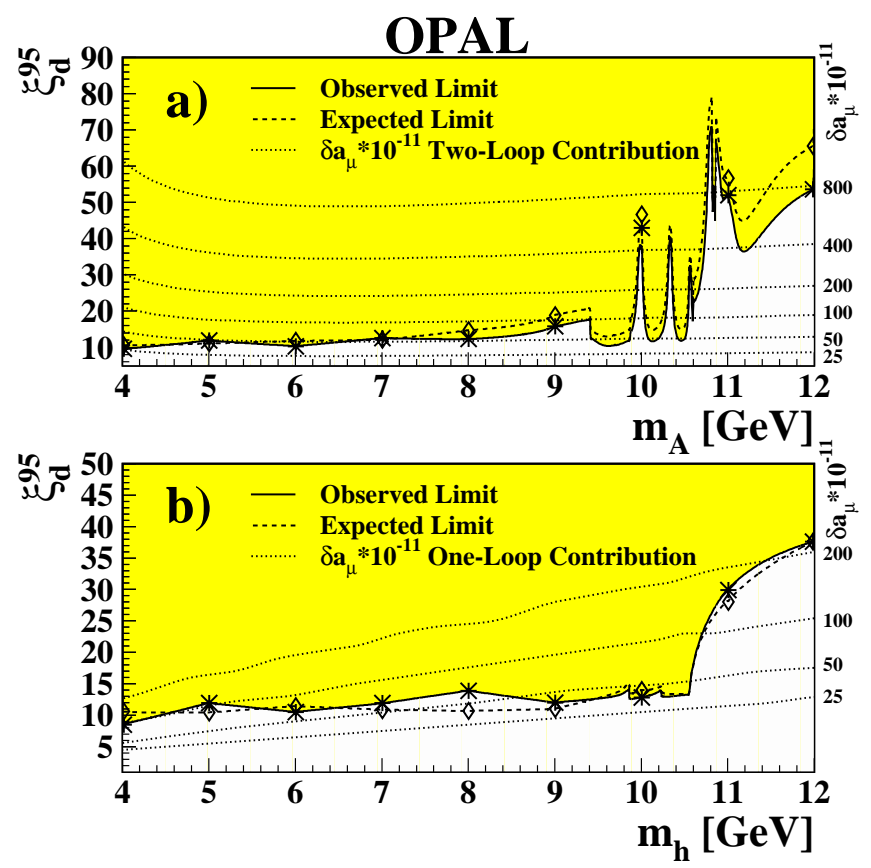

Figure 9: Excluded values of $\xi_{d}$ at 95\% C.L. in the 2HDM type II model with Standard Model particle content for the Yukawa production of a CP-odd $\mathrm{A}^{0}$ (upper plot) and for the CP-even $\mathrm{h}^{0}$

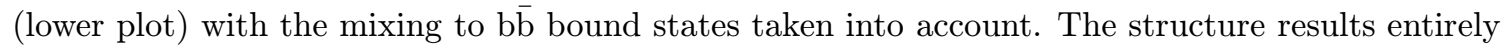
from the theoretically suggested branching ratios [2]- $\left.{ }^{-}\right]$. The expected (diamonds) and observed (stars) limits have been calculated at specific mass points ( $4-12 \mathrm{GeV}$ in one $\mathrm{GeV}$ steps) and linearly interpolated in between. The dotted lines are the contours of the predicted Higgs contribution

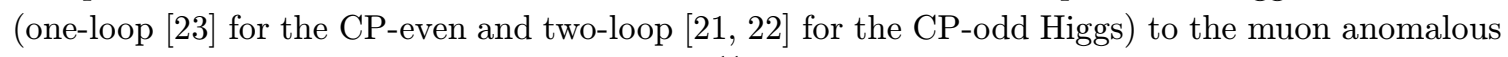
magnetic moment, $\delta a_{\mu}$ (Higgs) (in units of $10^{-11}$ ).

\section{References}

[1] J.F. Gunion, H.E Haber, G.L. Kane and S. Dawson, The Higgs Hunter's Guide, Addison-Wesley Publishing Company, 1990.

[2] Y. Okada, M. Yamaguchi and T. Yanagida, Prog. Theor. Phys. 85 (1991) 1.

[3] J. Ellis, G. Ridolfi and F. Zwirner, Phys. Lett. B257 (1991) 83.

[4] H.E. Haber and R. Hempfling. Phys. Rev. Lett. 66 (1991) 1815.

[5] M. Carena, J. R. Espinosa, M. Quirós and C.E.M. Wagner, Phys. Lett. B355 (1995) 209.

[6] M. Carena, M. Quirós and C.E.M. Wagner, Nucl. Phys. B461 (1996) 407.

[7] H.E. Haber, R. Hempfling and A.H. Hoang, Zeit für Phys. C75 (1997) 539.

[8] S. Heinemeyer, W. Hollik and G. Weiglein, Eur. Phys. Jour. C9 (1999) 343. 
[9] J. R. Espinosa and R. Zhang, Nucl. Phys. B586 (2000) 3.

[10] ALEPH Collaboration, A flavour independent search for the Higgsstrahlung process in e+ecollisions at centre-of-mass energies from 189 to $209 \mathrm{GeV}$, Contribution to the EPS conference in Budapest, Hungary, 2001, ALEPH 2001-021 CONF 2001-018.

[11] DELPHI Collaboration, Generalised search for hadronic decays of Higgs bosons with the DELPHI detector at LEP-2, Contribution to the EPS conference in Budapest, Hungary, 2001, DELPHI 2001-070 CONF 498.

[12] L3 Collaboration, Flavour Independent Search for Hadronically Decaying Higgs Boson in Higgs-strahlung Process at $\sqrt{s}$ up to $209 \mathrm{GeV}$, Contribution to the EPS conference in Budapest, Hungary, 2001, L3 Note 2693.

[13] OPAL Collaboration, Searches for Higgs Bosons in Extensions to the Standard Model in e+eCollisions at the Highest LEP Energies, OPAL Physics Note PN-472 (2001), OPAL Collaboration, Model Independent Searches for Scalar Bosons with the OPAL Detector at LEP, OPAL Physics Note PN-449 (2000), OPAL Collaboration, Eur. Phys. J. C18 (2001) 425-445.

[14] ALEPH, DELPHI, L3 and OPAL Collaborations, and the LEP Higgs Working Group, "Generalised Search for Hadronic Decays of Higgs Bosons at LEP-2", LEP Higgs WG Note 2001-07, July, 2001.

[15] P.H. Chankowski, M. Krawczyk et al., Eur. Phys. J C11 (1999) 661.

[16] OPAL Collaboration, G. Abbiendi et al., OPAL Physics Note PN475 (2001).

[17] S. Heinemeyer, W. Hollik and G. Weiglein, Phys. Rev. D58 (1998) 091701, Phys. Lett. B440 (1998) 296, hep-ph/9807423 and JHEP 0006 (2000) 009.

[18] ALEPH, DELPHI, L3 and OPAL Collaborations, and the LEP Higgs Working Group, "Searches for the Neutral Higgs Bosons of the MSSM: Preliminary Combined Results using LEP Data Collected at Energies up to 209 GeV", LHWG note 2001-02.

[19] J. Kalinowski and M. Krawczyk, Acta Phys. Polon. 27 (1996) 961.

[20] J.P. Leveille, Nucl. Phys. B137 (1978) 63.

[21] D. Chang, W.-F. Chang et al., Phys. Rev. D63 (2001) 091301; K. Cheung, C.-H. Chou et al., Muon Anomalous Magnetic Moment, Two-Higgs-Doublet Model, and Supersymmetry, hep-ph/0103183.

[22] M. Krawczyk, The new $(g-2)_{\mu}$ measurement and limit on the light Higgs bosons in 2HDM (II), hep-ph/0103223v3 (Submitted to Phys. Rev. D). .

[23] A. Dedes and H.E. Haber, JHEP 0105 (2001) 6.

[24] CUSB Collaboration, P. Franzini et al., Phys. Rev. D35 (1987) 2883.

[25] OPAL Collab., Search for Yukawa Production of a Light Neutral Higgs Boson at LEP, OPAL Physics Note PN483.

[26] M. Drees and K.-I. Hikasa, Phys. Rev. D41(1990) 1547. 